\title{
La Historia Oral en contextos digitales. Transformaciones recientes de un subcampo disciplinario
}

\author{
Juan Andrés Bresciano*
}

\section{Introducción}

En el transcurso de las últimas décadas, la aplicación de las tecnologías de la información y de la comunicación en la investigación histórica genera innovaciones significativas que afectan, prácticamente, a todas las instancias de producción, organización y divulgación del conocimiento con respecto al pasado. La Historia Oral, en cuanto subcampo disciplinario, no permanece ajena a esas transformaciones, en la medida en que ciertos cambios tecnológicos introducen modalidades originales de recabar, procesar, analizar y difundir testimonios orales, que inciden en el plano heurístico, hermenéutico, organizativo y discursivo de la propia especialización disciplinaria. Sin pretensión de exhaustividad, podrían indicarse las siguientes variaciones técnico-metodológicas como las más notorias:

(i) Los grabadores digitales modifican el modo en que las entrevistas se realizan al introducir una nueva clase de soporte que, por sus peculiaridades, habilita operaciones novedosas de análisis y de procesamiento de los registros.

\footnotetext{
* Profesor del Departamento de Historiología, Instituto de Ciencias Históricas, Facultad de Humanidades y Ciencias de la Educación, Universidad de la República, Uruguay.
} 
(ii) La creación de programas, de utilitarios y de aplicaciones informáticas destinados a planificar, implementar y procesar entrevistas repercute ampliamente en el desarrollo de los trabajos de campo.

(iii) La digitalización masiva de los registros orales y audiovisuales producidos en soportes analógicos abre las puertas a una utilización mucho más provechosa de fuentes que existían desde hace décadas, pero que resultaban de acceso restringido en razón del modo en que fueron generadas.

(iv) La conformación de repositorios digitales que conservan fuentes específicas para la Historia Oral supone una modalidad original de relacionamiento entre el investigador y los archivos, la cual redunda en una mayor eficiencia en las compulsas documentales.

(v) La generalización de portales telemáticos destinados a la divulgación de centros y de archivos que cultivan la oralidad estimula el acceso generalizado a cierta clase de fuentes orales y fomentan el intercambio académico más allá de fronteras geográficas.

(vi) La implementación de las tecnologías multimediales - que facilitan la integración de los componentes textuales, gráficos, orales y audiovisuales de un producto historiográfico en soporte electrónico propician nuevas formas de comunicar contenidos, que ya no se limitan a exposiciones de carácter lineal en las que prevalece la palabra escrita.

En este contexto de innovaciones, el presente artículo se plantea como objetivo general ofrecer una visión panorámica de las implicancias del impacto de los recursos digitales en el ejercicio de la Historia Oral - en todas y cada una de las etapas del proceso investigativo - a partir de la presentación de ciertos casos ilustrativos.

\section{El relevamiento telemático de fuentes testimoniales}

En todo proyecto de investigación en el que la oralidad constituya un componente heurístico ineludible, al investigador se le plantean dos opciones que no son necesariamente excluyentes: realizar entrevistas planificadas que le brindarán la información que precisa, o acudir a fuentes preexistentes. En este último caso, debe localizar documentos que registran tradiciones orales, narraciones o testimonios personales consignados en alguna clase de soporte 
sonoro o audiovisual, y surgidos de iniciativas vinculadas con el mundo académico o con entidades de la sociedad civil.

Un conjunto de factores tecnológicos y socioculturales estimulan, en décadas recientes, la producción y difusión de testimonios orales, en una escala y en una diversidad tipológica que no conoce precedentes. Entre los factores tecnológicos, cabría citar el abaratamiento de los dispositivos que posibilitan la grabación digital de la voz y de la imagen, así como la expansión vertiginosa de Internet y de las formas de comunicación de experiencias personales que fomenta. En lo que respecta a los factores socioculturales, habría que referir el desarrollo de una suerte de autoconsumo de masas, en razón del cual cientos de millones de personas se hallan propensas a producir registros de sus propias vidas - como parte de una sociedad del espectáculo - y a consumir los que producen sus congéneres, ya sean figuras conocidas o ciudadanos corrientes. También conviene tener presentes las iniciativas de organizaciones de la sociedad civil y de centros académicos que incentivan, en forma sistemática, la creación y la circulación telemática de relatos que dan cuenta de las más variadas realidades.

Como resultado de la confluencia de estos factores, emerge un universo heurístico de fuentes orales en formato digital que plantea desafíos novedosos para el historiador, puesto que debe aplicar criterios rigurosos para identificar, relevar, seleccionar y evaluar tales documentos. Sólo a modo indicativo, en esta segunda sección del artículo se referirán algunas de las variantes tipológicas que asumen las nuevas fuentes, así como los contextos en que se producen y consumen. En esta presentación somera, se transitará de las expresiones más espontáneas e individualistas que asumen el fenómeno hasta las más orgánicas y estructuradas.

Siguiendo el criterio enunciado, debe mencionarse, en primera instancia, el aporte de los relatos autobiográficos digitales y el de las bitácoras personales presentes en los blogs. ${ }^{1}$ La propia configuración de estos últimos favorece el registro cotidiano -ya sea textual, oral, audiovisual o gráfico de las experiencias que involucran a un individuo en particular, al tiempo que animan la producción de narrativas vitales. Aunque parezcan materiales heteróclitos, de escasa calidad literaria o de poca trascendencia histórica, constituyen, de hecho, fuentes de gran valor para el estudio de los fenómenos

1 Véase Elisabeth Lindsay (2009). 
históricos más dispares del tiempo presente - como las modalidades que asumen las migraciones actuales, los desafíos del multiculturalismo, las dinámicas de las identidades colectivas, o el impacto de las redes sociales en los movimientos contestatarios y antisistémicos.

Una segunda modalidad que adquiere una relevancia progresiva consiste en la creación sistemática de fuentes orales en cuanto insumos para las historias de familia. Tradicionalmente, esa clase de historias se asocia al estudio de las élites políticas, económicas y sociales, y se vincula con ciencias auxiliares tales como la Genealogía, la Nobiliaria y la Onomástica. Con la democratización paulatina del sujeto histórico, su uso se extiende más allá de los sectores privilegiados, y con las sucesivas renovaciones teórico-metodológicas que experimentan las Ciencias Históricas en el siglo XX, el estudio de las generaciones que integran una familia corriente resulta de utilidad para comprender la imbricación de los procesos macrosociales y los microsociales. Si bien las nuevas historias de familia constituyen el resultado de la práctica investigativa de historiadores, sociólogos y antropólogos, existen formas de cultivarla que se desprenden de la labor académica. La propia sociedad de autoconsumo de masas y la explosión de las redes sociales propician el interés personal por pesquisar - desde una perspectiva amateur - la historia de la familia a la que se pertenece, a partir de la papelería y de los relatos orales de los integrantes del núcleo familiar. De hecho, existen programas informáticos especialmente concebidos para que el ciudadano corriente construya su historia familiar, gracias a herramientas genealógicas y de fuentes orales en soporte digital. Tales programas auxilian en el proceso íntegro de relevar los datos, de procesarlos, de estructurarlos en diagramas y en formas expositivas estandarizadas, y de difundirlos a través de productos telemáticos. ${ }^{2}$

Un proceso semejante acontece con la Historia local y los testimonios orales. El estudio histórico de lo local se identifica, generalmente, con las expresiones más típicas de la Historiografía erudita del siglo XIX y de las primeras décadas del XX. En ciertos países, en particular los anglosajones, esas prácticas se manifiestan en sociedades o instituciones de historiadores no profesionales especializadas en recabar toda clase de documentos atinentes a la historia de la pequeña comunidad de la que forman parte. Al igual que la Historia familiar, en el último tercio del siglo pasado, la Historia local resurge como expresión de una Historia científico-social interesada en estudiar la

2 Véase http://familyoralhistory.us. 
autonomía de los espacios comunitarios en contextos de globalización, y el impacto de los procesos mundiales en el ámbito identitario y vivencial más inmediato. Sin embargo, la revolución tecnológica actual - que multiplica las formas en que se canaliza la información y se comunican los sujetos históricos - conduce a un nuevo florecimiento de las Historias locales desvinculadas de las dos formas anteriores. Se trata de una modalidad no profesional, que responde a iniciativas horizontales en su conformación y en su operativa, y multimediáticas en sus contenidos. Por tal razón, las propuestas en que se plasma, integran testimonios orales - junto con otra clase de insumos - y los publicitan amalgamados con otras clases de registros, sin ningún filtro metodológico que se emparente con la labor historiográfica académica. ${ }^{3}$

La última modalidad a referir en esta caracterización somera consiste en los proyectos de relevamiento y sistematización de fuentes orales para uso público en portales telemáticos, como ocurre con el sitio Web denominado In the First Person. ${ }^{4}$ Este sitio recopila miles de narrativas personales en idioma inglés, entre las que figuran cartas, diarios, memorias, autobiografías, entrevistas y relatos orales. Todos sus materiales testimonian, en primera persona, los avatares de la existencia de sus autores, y brindan a los cientistas sociales un universo de relatos personales sobre las trayectorias de hombres y mujeres, de actores relevantes y de ciudadanos corrientes, de patrones y de trabajadores, de adultos y de jóvenes, de todas las procedencias geográficas y de los más variados orígenes étnicos. Para cumplir con ese fin, In the First Person se sirve de programas capaces de localizar automáticamente aquellos sitios Web que se ajustan a los criterios del proyecto. También acude a materiales que se hayan editado en formatos más tradicionales (libros, revistas, folletos, etc.). Una vez que identifica los relatos y obtiene la autorización (si corresponde) para hacer uso de ellos, procede a su reproducción digital, acompañada en algunos casos de la transcripción de sus contenidos. Conseguidas las fuentes, se las clasifica utilizando hasta veinte tipos distintos de vocabularios controlados. Como fruto de este proceso, se genera una base de datos que cualquier usuario registrado puede emplear. Si el consultante logra localizar una o varias narraciones de su interés, accede directamente a ellas para reproducirlas, en caso de que no existan restricciones.

3 Véase, a modo de ejemplo, http://www2.pgohg.org:8080.

4 Véase http://www.inthefirstperson.com/firp/index.shtml. 


\section{La producción y análisis de fuentes orales mediante dispositivos y programas informáticos}

En los últimos quince años, los programas que auxilian al historiador en sus trabajos de campo han ampliado notablemente la capacidad de producir fuentes, de procesarlas y de organizarlas de manera eficiente. Gracias a las nuevas tecnologías, el historiador puede generar documentos sonoros y audiovisuales de alta calidad, con dispositivos externos a un ordenador, e incorporar y almacenar múltiples clases de registros en un mismo utilitario, sin importar su naturaleza o su formato. A esto se agrega la posibilidad de estructurar, codificar y analizar tales insumos, de acuerdo a un amplio espectro de operaciones. En tal sentido, los aportes tecnológicos al ejercicio de la Historia Oral responden a dos variantes:

(i) La de los dispositivos y programas que permiten el registro y el procesamiento digital de las entrevistas para producir documentos.

(ii) La de las aplicaciones destinadas a la transcripción y edición de las fuentes propiamente dichas.

En lo que respecta a las cuestiones estrictamente técnicas del registro digital de entrevistas, existen tres factores a tener en cuenta. El primero de ellos consiste en la elección del dispositivo que se utilizará para crear la fuente oral. Actualmente se emplean cuatro tipos de recursos que auxilian al investigador en esa tarea:

(i) Los grabadores de memoria flash, que resultan muy prácticos y relativamente baratos.

(ii) Los grabadores con disco duro, que habilitan registros extensos, de alta calidad y fidelidad, y que simplifican la transferencia de los archivos a rápida velocidad.

(iii) Los grabadores con disco compacto, que tienen notorios límites en cuanto capacidad de almacenamiento, pero que se presentan como una opción económica.

(iv) Los ordenadores con dispositivos de grabación y con micrófono interno, caracterizados por la alta calidad de sus registros y por su amplia capacidad de memoria (Boyd, [s.d.]). 
El segundo factor que debe considerarse en la grabación digital - además de la elección del dispositivo - es el tipo de registro de la entrevista, que admite dos opciones: monoaural o estéreo. En términos generales, resulta muy útil la grabación en estéreo, ya que permite aislar un canal para la voz del entrevistador y el otro para la del entrevistado. Simplifica, de este modo, la transcripción de la entrevista, ya que es posible diferenciar ambas voces cuando se superponen en algunos segmentos de la grabación (Boyd, [(s.d.]).

El tercer y último factor se relaciona con los formatos de los archivos de audio. Cada formato presenta ventajas e inconvenientes que el historiador y el archivólogo deben tener en cuenta. Para los registros no comprimidos, los formatos más habituales suelen ser Wave y AIFF. Los grabadores portátiles de trabajo de campo emplean el primero, mientras que el segundo se asocia con las aplicaciones de las computadoras Mac. De ambos formatos, el más usual es el Wave, por ventajas tales como la incorporación de metadatos en el propio registro digital. En lo que respecta a los formatos comprimidos, cabía mencionar el de los archivos MPEG, utilizado con mucha frecuencia cuando se desea divulgar un documento a través de Internet (Boyd, [(s.d.]).

Además de ponderar cuidadosamente estos tres factores, es preciso seguir algunas pautas técnicas complementarias con respecto al registro digital de las entrevistas, si se procura garantizar la fidelidad del documento creado. Entre las pautas más generales, sobresalen las siguientes:

(i) Se debe realizar una cuidadosa evaluación del dispositivo de registro elegido, en función de los objetivos del proyecto. En ciertas ocasiones, se tiende a economizar en la compra de aparatos que no generan fuentes de calidad, mientras que con un gasto adicional podría obtenerse una herramienta que produce archivos claramente audibles, sin interferencias, inconsistencias o ruidos que posteriormente dificulten su análisis.

(ii) Conviene utilizar un micrófono externo que asegure la calidad de la fuente obtenida. Los micrófonos incorporados al computador no facilitan un mejor registro, porque deben aproximarse a la fuente de emisión de voz, lo cual plantea dificultades prácticas cuando se trata de una entrevista.

(iii) Es recomendable efectuar la grabación en archivos WAV no comprimidos con un mínimo de calidad de 16-bit/44.1KHz. 
(iv) Se aconseja desconectar los teléfonos celulares durante la entrevista, porque aunque se encuentren en modo vibrador, las señales que emite son capaces de interferir con la grabación y afectar su calidad (Boyd, [(s.d.]).

Como se indicó oportunamente, el aporte de las tecnologías recientes no se circunscribe al registro de fuentes orales. De hecho, los programas informáticos destinados al desarrollo y procesamiento de tales fuentes se cuentan por decenas. Aunque algunos ofrecen funciones similares, los que apuntan a la filmación de la entrevista presentan perfiles diferenciados. A modo de ejemplo podría referirse un utilitario como Theme, ${ }^{5}$ que se centra en la producción de registros audiovisuales, obtenidos a partir de una plataforma estática (desde la cual se captan gestos, posturas, movimientos, posiciones, expresiones faciales de los sujetos que participan de la acción). Theme habilita el mismo tipo de registros en diversas clases de ámbitos y espacios, en los que la movilidad del observador constituye un factor básico. En contraste, un programa como OCS Tools ${ }^{6}$ se especializa en la estructuración de la fuente que crea, mediante la utilización de macros que ejecutan rutinas de codificación y descripción de los hechos al mismo tiempo que éstos acontecen.

Las entrevistas generan documentación científica que suele archivarse para que la utilicen otros investigadores. El texto del cuestionario en que se basan, las fichas técnicas que la identifica, el registro de audio (o de video) en que se consignan, las notas de observación que las acompañan, así como los en que se las desgraba, constituyen clara prueba de ello. Las nuevas tecnologías proporcionan numerosos medios para la creación de esta clase de productos, según la entrevista se realice personalmente, en forma telefónica, o acudiendo al correo electrónico. Para las entrevistas que se basan en preguntas cerradas, un programa como IT-CAPI [Computer Assisted Personal Interview] permite: (i) el diseño de diferentes modelos de cuestionarios; (ii) la especificación del tipo de entrevista a realizar y del tipo de preguntas (codificadas, numéricas, textuales, abiertas, de múltiples opciones, etc.); (iii) la edición permanente de los datos que se colectan, inclusive durante el desarrollo de las entrevistas. ${ }^{?}$

5 Véase http://www.noldus.com/human-behavior-research/products/theme.

6 Véase http://www.trctech.com.

7 Véase http://www.interviewtechnology.com/desan/framemain/index.htm?../submenu/products/ capi.htm. 
Para las entrevistas que se basan en preguntas abiertas, un utilitario como Freerecorder ${ }^{8}$ - entre tantos otros - habilita el registro digital de entrevistas desde un PC, la selección de fuentes de sonido, la detención y reanudación automática de una grabación, el almacenamiento de los registros en archivos MP3, etc. Algo semejante acontece con PolderbitS Sound Recorder and Editor, ${ }^{9}$ ya que posibilita la grabación digital de entrevistas desde una computadora portátil, su almacenamiento en un archivo digital de audio, la reproducción de las entrevistas realizadas, la edición de cada una de ellas, la organización de sus segmentos, y la remisión por correo electrónico a cualquier destinatario de los archivos de audio en formato MP3.

Tanto para entrevistas abiertas como cerradas existen recursos informáticos que procesan las fuentes primarias obtenidas - el registro de audio - y las convierten en registros textuales. Dentro de ciertos límites, son capaces de llevar a cabo transcripciones automáticas de entrevistas enteras, de conferencias, etc. Asimismo, constituyen un instrumento auxiliar para el registro de las observaciones, impresiones o ideas del investigador, que luego se reproducen textualmente. El programa Transcriber (Boudahmane et al., [s.d.]) ofrece herramientas para la reproducción textual del contenido de archivos de audio, y brinda asistencia al proceso de anotación manual de signos de discurso. Asimismo, habilita la segmentación de grabaciones extensas y la identificación y categorización de giros, cambios temáticos y condiciones acústicas de los registros. Por su parte, OpenSpeechRecognizer ${ }^{10}$ puede reconocer frases y oraciones completas a partir de un archivo de audio, es capaz de distinguir voces con diferentes acentos y en contextos de interferencia acústica, y logra amoldarse a los patrones discursivos de los hablantes.

En base a los servicios que aportan los programas reseñados, puede concluirse que quien desarrolla trabajos de campo en el ámbito de la Historia Oral a principios del siglo XXI se beneficia de una amplia gama de medios tecnológicos, que complejizan su labor (ya que le obligan a adoptar nuevas clases de determinaciones técnico-metodológicas), pero le confieren instrumentos de preservación documental y de análisis heurístico que se multiplican y diversifican día a día.

8 Véase http://www.applian.com/freecorder4.

9 Véase http://www.polderbits.com.

10 Véase http://www.nuance.com/recognizer/openspeechrecognizer. 


\section{El desarrollo de una archivística histórica vinculada con los registros orales digitales}

En las investigaciones sobre el pasado y en la tarea de preservación del patrimonio documental de una comunidad, los archivos cumplen una función primordial. Esa función adquiere una renovada vigencia ante los desafíos que conlleva el advenimiento de una sociedad de la información y del conocimiento. En lo que atañe a los archivos históricos de fuentes orales, la progresiva digitalización de los soportes plantea problemas inéditos en actividades tales como la preservación de los documentos, la clasificación y descripción de sus contenidos, y la puesta en servicio de los colecciones.

Los registros de audio y video suelen ser voluminosos - si se los compara con los documentos producidos en procesadores de textos - y dado que crecen en forma exponencial, motivan un problema serio de almacenamiento. Por otra parte, la obsolescencia de equipos y de programas informáticos una tendencia que se acentúa a medida que la Revolución Informática se acelera - crea serios inconvenientes para una adecuada conservación de los documentos en sus soportes originales, puesto que desparecen paulatinamente los equipos que permiten su lectura. La migración de registros a los nuevos soportes constituye un paliativo necesario, pero no plenamente satisfactorio, sobre todo cuando los recursos materiales de los repositorios son limitados y no garantizan el mantenimiento de los ordenadores y programas de décadas anteriores (Archiving, 2011).

Un problema equiparable lo suscitan aquellos documentos orales cuyo soporte original es análogo y no digital. No resulta sencillo establecer parámetros universales para la preservación de tales de fuentes, o la de diseñar proyectos archivísticos para su digitalización masiva, puesto que no existen consensos definitivos con respecto en qué clase de formatos deberían conservarse. El archivólogo debe ponderar muy bien sus determinaciones y considerar detenidamente cada caso, para garantizar la mayor fidelidad posible en la conservación de fuentes cuyos contenidos migran de un soporte análogo a otro digital.

La seguridad requerida en el almacenamiento de los documentos también genera problemas logísticos. Dado que es conveniente disponer de varias copias de un registro - en emplazamientos distintos, por si acontece un siniestro - se produce, como resultado, una demanda adicional de espacio 
destinado a las tareas de preservación, que no todos los repositorios se encuentran en condiciones de satisfacer adecuadamente.

La labor archivística no se restringe a la mera conservación de materiales. Para que el usuario pueda consultarlos, es preciso contar con instrumentos de descripción documental adecuados. En materia de registros orales digitales, la descripción asume funciones que superan ampliamente el horizonte de las que tradicionalmente se realizaban. Actualmente, es indispensable consignar una amplia serie de metadatos, entre los cuales se incluyen los relativos a:

(i) Los programas de consulta de las fuentes orales almacenadas.

(ii) El formato específico de los tipos documentales.

(iii) La estructura de los registros, en lo que atañe a forma y contenidos.

(iv) La procedencia de las fuentes, con indicación de los equipos y utilitarios que se emplearon para crearlas.

(v) La validación y autenticación de cada documento, sobre todo si ha sido objeto de traslado o de migración de datos.

(vi) Los derechos de autor que afectan a los registros, en el caso de grabaciones de entrevistas. (Archiving, 2011).

Este conjunto de metadatos contribuye a garantizar la integridad, la accesibilidad y la eficaz utilización de los fondos de archivos orales en soporte digital, más allá de las variaciones que los cambios tecnológicos introducen regularmente. El celo con que se cumple dicho objetivo lleva a que ciertos repositorios incluyan metadatos altamente específicos sobre los contenidos de una selección de fuentes orales o sobre la totalidad de ellas. A su vez, la generación de un listado exhaustivo de descriptores para clasificar cada documento, permite que el usuario interesado en un tema acotado identifique automáticamente todos aquellos registros que aportan alguna referencia.

La eficacia de la descripción documental se potencia cuando la puesta en servicio de los documentos reduce al mínimo el tiempo que media entre la localización de un registro y su consulta. Por ello, el acceso telemático a los fondos de un repositorio oral se convierte en una transformación revolucionaria en el campo de las investigaciones históricas. Los portales de Internet de los principales archivos orales del planeta ponen a disposición de cualquier usuario nacional o extranjero el cuadro clasificatorio de sus fondos, y ofrecen un acceso (condicional) a un porcentaje determinado de los documentos que custodian. Este acceso condicional - que depende de una suscripción 
institucional o del pago de derechos - se presenta como un inconveniente menor, si se ponderan los efectos altamente positivos de la utilización y reproducción a distancia de un ingente volumen de registros orales. Por otra parte, es preciso tener en cuenta la tendencia mundial a ampliar el número de documentos identificables y/o consultables en línea.

Corrobora esa tendencia los servicios que brindan The British Library Sound Archive, ${ }^{11}$ sus Oral History Collection, y sus National Life Stories, cuya documentación puede relevarse a través de catálogos disponibles en el sitio Web institucional. En escala más reducida, pero con acceso directo a las transcripciones de las fuentes originales, The Rutgers Oral History Archive ${ }^{12}$ también ilustra las ventajas del acceso telemático, en este caso, para los interesados en estudio de testimonios orales de veteranos estadounidenses de la Segunda Guerra Mundial, la Guerra de Corea, la Guerra de Vietnam y la Guerra Fría. Con un grado mayor de sofisticación tecnológica, The History Makers Digital Archive ${ }^{13}$ proporciona acceso restringido a las grabaciones audiovisuales de cientos de entrevistas a figuras protagónicas de la comunidad afrodescendiente de Estados Unidos. Si se cumplen los requisitos de suscripción, es posible consultar, además, los documentos audiovisuales mediante Internet, los cuales se encuentran clasificados en múltiples categorías, y descriptos de manera minuciosa.

\section{El nacimiento de redes, de centros y de proyectos colectivos destinados al cultivo de la Historia Oral Digital}

La institucionalización académica configura uno de los ámbitos en los que el influjo de las nuevas tecnologías en la investigación histórica resulta claramente perceptible. En el transcurso de los últimos veinte años, surgen al menos treinta centros universitarios que desde Estados Unidos y Europa se constituyen en modelo de la adopción sistemática de los recursos informáticos en los estudios del pasado. Estos centros estimulan una Historiografía plenamente digital, desde las tareas de recabar la documentación necesaria

11 Véase http://www.bl.uk/nsa.

12 Véase http://oralhistory.rutgers.edu.

13 Véase http://www.idvl.org/thehistorymakers/Home.html. 
para el abordaje de un tema, hasta la instancia de difusión de los resultados investigativos. La Historia Oral, en cuanto subcampo disciplinario, no es una excepción en dicha tendencia. En los últimos años, se consolidan tres modalidades de institucionalización académica que reflejan las nuevas dinámicas. La primera se relaciona con la conformación de redes telemáticas de discusión y de intercambio entre especialistas. La segunda se vincula con la conformación de centros, propiamente dichos, entendidos como unidades operativas de instituciones universitarias o terciarias, en los que se ejerce la docencia, la investigación y las tareas de extensión. La tercera modalidad supone el emprendimiento de proyectos colectivos - que involucran a varias entidades y organizaciones - y que indagan sobre la Historia Oral Digital, sus posibilidades presentes y su proyección hacia el futuro.

Un ejemplo representativo de la primera modalidad procede de la $H$ Oralhist. ${ }^{14}$ Afiliada a la Oral History Association, esta red promueve la discusión de cuestiones relativas a la creación de registros digitales y a la digitalización de fuentes en soporte analógico. Su portal contiene enlaces a los sitios Web de los principales repositorios orales y colecciones, así como a centros que fomentan esta clase de estudios y programas de investigación que los aplican. Posee, además, un archivo de las discusiones desarrolladas en red, así como una guía de preguntas y respuestas a las cuestiones fundamentales de la Historia Oral Digital.

Ejemplos de la segunda modalidad se cuentan por decenas. Solo a modo ilustrativo podrían indicarse cinco de ellos que evidencian la diversidad organizativa predominante:

(i) El Institute for Oral History, de la Baylor University, ${ }^{15}$ y su taller en línea para quienes comienzan a familiarizarse con las técnicas de la entrevista y de la recopilación de fuentes orales. Su archivo incorpora más de ochocientas memorias orales de acceso telemático gratuito. Algunos de sus contenidos han sido transcriptos sistemáticamente, aunque su indización aún es parcial.

14 Véase http://www.h-net.org/ oralhist.

15 Véase http://www.baylor.edu/oralhistory. 
(ii) El Centre for History \& Digital Storytelling, de la Concordia University, ${ }^{16}$ tiene por objetivo contribuir a la cooperación en materia de investigación y enseñanza en el campo de la Historiografía Digital. Trabaja no sólo con el cuerpo docente y estudiantil de la institución referida, sino con otras comunidades académicas, locales y extranjeras. El centro dispone de variados recursos para el registro de fuentes orales y su digitalización. Entre los proyectos específicos que lleva cabo, figura el desarrollo de herramientas informáticas aplicadas a la producción de notas orales que se agreguen de manera automática a los documentos.

(iii) El Centro Talking History, ${ }^{17}$ de la University at Albany/University of the State of New York, se dedica a la producción, distribución y enseñanza de toda clase de fuentes históricas basadas en la oralidad. Proporciona a investigadores, docentes, estudiantes y público en general colecciones que incluyen documentales orales, discursos, conferencias, debates, historias y relatos orales, etc.

(iv) El Louie B. Nunn Center for Oral History, de la University of Kentucky Libraries, ${ }^{18}$ impulsa un sistema que se denomina Oral History Metadata Synchonizer. Consiste en una herramienta sumamente eficiente para identificar y acceder a fuentes orales disponibles en línea. El sistema dispone de instrumentos de búsqueda que, a través de descriptores, localizan contenidos de documentos indizados y transcriptos en diferentes sitios Webs.

(v) El Center for History and New Media, ${ }^{19}$ establecido en 1994 en la George Mason University, aplica las tecnologías de la información y la comunicación en la tarea de democratizar el conocimiento histórico. En un esfuerzo sistemático por ampliar el público destinarlo del saber disciplinario, se interesa por la incorporación de voces múltiples y estimula la participación de los ciudadanos corrientes en la preservación de fuentes relacionadas con el estudio del pasado. Los recursos digitales que pone a disposición de los usuarios se aplican a los métodos y técnicas de la investigación científico-social, incluida la Historia Oral. Destinados a académicos, educadores, estudiantes y

16 Véase http://storytelling.concordia.ca.

17 Véase http://www.talkinghistory.org.

18 Véase http://uky.edu/libraries/nuncenter.

19 Véase http://chnm.gmu.edu. 
potenciales interesados en ese campo, tales recursos se emplean no sólo en la producción de fuentes orales, sino en la incorporación de estas últimas en productos historiográficos articulados a partir de un formato mutimediático.

En lo que respecta a proyectos colectivos focalizados en el estudio de la Historia Oral Digital, existe un emprendimiento interdisciplinario que amerita una presentación detallada. Denominado Oral History in the Digital Age, ${ }^{20}$ nace de la confluencia de aportes del Michigan State University Museum y la Oral History Association, entre otras entidades. El proyecto se propone algunos objetivos estratégicos:

(i) Implementar grupos de trabajo que discutan los problemas básicos que enfrenta la Historia Oral Digital.

(ii) Desarrollar pautas técnicas normalizadas para el ejercicio de la Historia Oral basada en registros audiovisuales.

(iii) Crear un portal para el intercambio de experiencias académicas en ese campo.

(iv) Promover redes que vinculen a los investigadores con los principales repositorios documentales, para la compulsa de información y la divulgación de fuentes poco utilizadas.

Los grupos de trabajo constituidos a partir del proyecto se dedican al análisis de siete temas que pautan la tónica de las discusiones actuales:

(i) La propiedad intelectual de los registros surgidos de entrevistas, en particular aquellos que surgen en un contexto predigital. Se trata de definir criterios que resulten útiles para los investigadores, las instituciones y los funcionarios de repositorios públicos y privados.

(ii) La transcripción de los registros orales/audiovisuales. Sin lugar a dudas, esta operación se encuentra sujeta a revisión, en la medida en que las nuevas tecnologías permiten un acceso codificado y automático a tramos específicos de un testimonio almacenado en un soporte digital, así como su inmediata reproducción sonora. Aun así, la cuestión de

20 Véase http://projects.matrix.msu.edu/ohda/grant/. 
la descripción de las piezas de una colección y una indización de sus contenidos suponen un desafío que los medios actuales resuelven con mayor eficiencia.

(iii) El registro audiovisual de los testimonios. El abaratamiento de las cámaras digitales de video estimula y generaliza la filmación de la entrevistas. De este modo, se abre un nuevo campo de análisis, que incluye la comunicación no verbal en el transcurso de una entrevista, así como los elementos contextuales relativos al espacio y el entorno. Por otra parte, se simplifica la indización multidimensional de la documentación generada, y su propia divulgación, ya que existe un público ávido de consultar esta clase de productos.

(iv) Los cambios tecnológicos que inciden en el ejercicio de la Historia Oral. El estudio de sus aplicaciones se centra en cuatro instancias: el trabajo de campo, la digitalización de los documentos, el acceso a los materiales producidos y la creación de herramientas informáticas de consulta de las fuentes almacenadas.

(v) Los fundamentos académicos de las prácticas de Historia Oral Digital. Dado que las nuevas tecnologías modifican las relaciones entre los investigadores y los repositorios que utilizan, es conveniente implementar normas que regulen la preservación de los fondos de repositorios institucionales y colecciones privadas, y el respeto a la propiedad intelectual en contextos digitales.

(vi) La consulta de los registros electrónicos y las interfases que lo hacen posible. El uso de los testimonios orales asume formas muy distintas a las tradicionales, ya que las aplicaciones informáticas incluyen búsquedas no lineales de contenidos, y posibilitan la asociación de fuentes con productos historiográficos, a través de enlaces hipermediales. En consecuencia, es preciso reflexionar sobre los efectos que estos instrumentos generan en la lectura/audición/observación de los documentales. Todo ello requiere considerar las interfases computacionales, las clases de usuarios que utilizan los materiales y los propósitos que persiguen. ${ }^{21}$

21 Véase http://projects.matrix.msu.edu/ohda/grant/. 


\section{La incorporación de la oralidad en un discurso historiográfico multimediático}

Las nuevas tecnologías inciden en la implementación de las investigaciones históricas, pero también gravitan sobre la configuración del propio discurso historiográfico. Antes de la generalización de los procesadores de textos, los programas computacionales y las redes telemáticas, los historiadores generaban productos que obedecían a una estructura relativamente homogénea. En ellos, el texto ejercía una supremacía indiscutible, y los materiales complementarios que se incorporaban - gráficos o iconográficos cumplían una función ilustrativa o demostrativa, siempre subordinada a la palabra escrita. Con la utilización de los recursos digitales en la producción de textos, el discurso historiográfico amplía sus posibilidades comunicativas y experimenta modificaciones en su propia naturaleza. Tales cambios se procesan en planos de complejidad progresiva.

En el plano más elemental, se encuentran las investigaciones basadas en los métodos y técnicas de la Historia Oral, cuyos resultados se comunican, básicamente, a través de la palabra escrita. Las entrevistas utilizadas como fuentes, en su formato original, se anexan al cuerpo central del producto, acompañadas (si es necesario) de las correspondientes transcripciones. Por lo tanto, el trabajo consiste en un archivo de texto con archivos de audio o archivos de video que se le adjuntan.

En un plano intermedio, existe otra clase de productos. Se trata de obras historiográficas multimediáticas en las que el texto, aún cuando constituye el pilar en que reposa la totalidad del trabajo, remite, mediante enlaces internos automáticos, a gráficas, a imágenes, o a segmentos de un archivo de audio o de video. Los enlaces hipermediales permiten transitar de un medio de información a otro, de modo tal que el lector es capaz de relacionar la interpretación que un texto le brinda con los datos que surgen de un tramo específico de una entrevista adjunta. Los materiales orales o audiovisuales - convenientemente presentados - adquieren una preponderancia creciente, y se autonomizan hasta cierto punto. Por la propia configuración de esta clase de trabajos, no existe una única secuencia de lectura, dado que el usuario consulta el producto en el orden que le dicta su interés, desplazándose, gracias a los enlaces, en un universo de textos, imágenes, registros orales y audiovisuales. En esta instancia, la oralidad no constituye una fuente que se torna útil gracias a la acción mediadora de las transcripciones textuales, sino que deviene un medio 
legítimo de comunicación de datos y de experiencias, que en su conjunto enriquecen y profundizan la intelección del tema abordado. Productos historiográficos de tal naturaleza suelen divulgarse en ediciones telemáticas de portales académicos de Internet. Un buen ejemplo lo proporciona el artículo de Robert Darnton (2000) titulado "An Early Information Society: New and Media in the Eighteenth-Century Paris". Este texto electrónico despliega enlaces a los mapas digitalizados de París, y a través de ellos se accede a toda clase de materiales: la cartografía de los cafés en los que la policía recogía información sobre actividades políticas, los informes elaborados por la policía, archivos de audio con canciones de contenido político, y las transcripciones correspondientes de todas ellas.

Finamente, existen productos de investigación que se transforman en repositorios virtuales capaces de integrar a todos los tipos básicos de fuentes históricas. En razón del tema del proyecto en que se basan, recopilan la documentación disponible en sus formatos originales, la digitalizan, y la organizan en fondos que sólo existen en el ciberespacio. Por lo tanto, sus contenidos se divulgan desde una plataforma telemática en la que se almacena la totalidad de los registros relevados, o a una selección de ellos. El proyecto Race \& Place. An African American Community in the Jim Crow South ${ }^{22}$ constituye un modelo paradigmático de tales repositorios, puesto que presenta, en fondos separados, las reproducciones digitales de documentos inéditos, de fuentes hemerográficas, de fuentes cartográficas, de fuentes iconográficas y de fuentes orales. En lo que atañe a estas últimas, incluye decenas de entrevistas en archivos de audio, con las correspondientes fichas descriptivas. Otro ejemplo notable que podría referirse es el del The September 11 Digital Archive ${ }^{23}$ Con más de ciento cincuenta mil piezas disponibles en línea, conjuga las más variadas fuentes para la historia de los atentados terroristas que destruyeron los Torres Gemelas. Estructurado en siete fondos, almacena documentos de textos, correos electrónicos, relatos, imágenes, animaciones digitales, entrevistas y registros de audio y video. Incluye también una sección con colecciones especiales, entre las que figuran, una titulada Ground One. Voices from Post-911 Chinatown. Esta última recoge treinta entrevistas a residentes de Chinatown, que fueron testigos de los atentados y de sus consecuencias. ${ }^{24}$

22 Véase http://www2.vcdh.virginia.edu/afam/raceandplace/oralhistory_main.html.

23 Véase http://911digitalarchive.org/index.php.

24 Véase http://911digitalarchive.org/chinatown/. 


\section{La utilización de las nuevas tecnologías en la enseñanza de la Historia Oral}

En su artículo "Digital History in the History/Social Studies Classroom", John K. Lee (2002) formula una serie de apreciaciones que merecen citarse:

Beyond the electronic storage and presentation of historical materials, digital history is also about the construction of narratives and the presentation of historical research findings. In the digital genre of history, students stand side by side with professional historians generating an infinite number of interpretations from the electronic archives of the Web. Digital history encourages a view of the past that is tentative and process orientated. In addition, the Web's hypertexuality encourages alternative narrative forms. Historian Edward Ayers has suggested that the nonlinear complexity supported by the Web is a means to deal more effectively with the multiple sequences, voices, outcomes and implications of historical narrative. Students writing historical narratives in hypertext will have the ability, through the construction of links, to exercise a greater sense of control over the narrative and particularly the structure of arguments within the narrative.

Los efectos de las nuevas tecnologías en la enseñanza de la Historia que señala Lee se reflejan claramente en una serie de iniciativas que cristalizan en tiempos recientes. El Comité de Educación de la Oral History Association reconoce la relevancia de los medios digitales en la enseñanza de la Historia Oral en el nivel secundario y terciario, y de hecho patrocina decenas de proyectos impulsados por estudiantes preuniversitarios y por sus respectivos docentes. ${ }^{25}$ Los participantes de tales propuestas realizan entrevistas, las sistematizan en repositorios digitales y las divulgan en portales de Internet. De este modo, a través del aprendizaje práctico de las técnicas de la Historia Oral, contribuyen a generar fuentes orales sumamente valiosas para el estudio de temas de alto grado de especificidad. Las iniciativas que promueve la asociación incursionan en numerosos tópicos de la Historia de Estados Unidos, tales como las narrativas de esclavos, las memorias de veteranos de guerra,

25 Véase http://www.oralhistory.org/wiki/index.php/Education_Committee. 
los testimonios del movimiento sufragista, las vivencias de protagonistas del movimiento por los derechos civiles durante los años 60, entre muchos otros.

Existen ejemplos de programas que comprometen directamente a los estudiantes liceales con el desafío de aplicar los métodos de la Historia Oral. The Urban School of San Francisco, liceo privado que se funda en 1966, desarrolla un curso -Telling Their Stories (TTS) ${ }^{26}$ - en el que los estudiantes entrevistan a residentes del Área de la Bahía involucrados en algún acontecimiento fundamental del siglo XX. Se trata de una instancia de formación curricular basada en el trabajo directo con fuentes primarias, que conlleva una tarea de extensión y de proyección social. Los alumnos participan en la selección de los entrevistados, en la realización de las entrevistas, en la transcripción y edición de los registros, y en el uso de los equipos digitales.

Otro ejemplo significativo lo aporta el proyecto What did you do in the War, Grandma? An Oral History of Rhode Island Women Turing World War II, realizado por estudiantes del South Kingstown High School. ${ }^{27}$ Los alumnos que participaron de la propuesta llevaron a cabo treinta y seis entrevistas a mujeres de su comunidad que eran jóvenes durante la Segunda Guerra Mundial. Veintiséis de esas entrevistas se transcriben e indizan en el sitio Web del proyecto, y en algunos casos se incluye la grabación digital con una descripción sumaria de sus contenidos.

Mediante iniciativas de esta índole, se cumplen tres objetivos que la Oral History Association define como primordiales:

(i) Involucrar a los estudiantes en la tarea de crear y procesar fuentes orales primarias, en cuanto instancia formativa por excelencia.

(ii) Relacionar a esos estudiantes con la comunidad de la que forman parte, al contribuir - merced al trabajo de campo y la recopilación de testimonios - con la conservación de la memoria histórica de determinados grupos o colectivos.

(iii) Concientizar a los alumnos del potencial de la Historia Oral como estrategia investigativa y de las dificultades técnico-metodológicas de su implementación en contextos específicos. ${ }^{28}$

26 Véase http://www.tellingstories.org.

27 Véase http://www.stg.brown.edu/projects/WWII_Women/.

28 Véase http://www.oralhistory.org/wiki/index.php/Education_Committee. 


\section{Conclusiones}

Las tecnologías de la información y de la comunicación ejercen un estímulo altamente positivo en un cultivo renovado de la Historia Oral. En lo inmediato, fomentan la ampliación de su campo de estudio, ya que la sociedad de la información estimula de manera superlativa la generación de testimonios orales y audiovisuales. A su vez, la literatura testimonial producida y difundida en redes telemáticas da lugar a una memorialística digital que los investigadores actuales no pueden obviar.

En lo que respecta a la operativa del historiador oral, los cambios que experimentan los métodos de la entrevista afectan no sólo a la naturaleza del registro obtenido, sino a las formas del procesamiento de sus contenidos. Transformaciones no menos sustanciales comprometen a los modos en que se almacenan, consultan y difunden tales fuentes.

Finalmente, la Historia Oral también se renueva en el plano de la organización e institucionalización académica - al surgir redes y centros que se interesan por la aplicación de los recursos digitales - y se transforma en la comunicación del conocimiento mismo, ya sea en lo que atañe a la naturaleza y estructura del discurso historiográfico, como a las prácticas de enseñanza y divulgación de un conocimiento del pasado que apela a la oralidad.

Por lo expuesto, no cabe duda que la Historia Oral, en cuanto subcampo disciplinario, atraviesa por una fase de renovación de sus prácticas técnico-metodológicos y de ampliación de su espectro heurístico, que aún dista mucho de haberse agotado y que sin duda se profundizará en el transcurso de los años por venir.

\section{Referencias}

ARCHIVING. 2011. Disponible en: <http://www.baylor.edu/content/services/ document.php/79807.pdf>. Acceso: 31 jul. 2011.

BOUDAHMANE, K. et al. Transcriber, a tool for segmenting, labeling and transcribing speech: presentation. ([s.d.]). Disponible en: <http://trans.sourceforge.net/en/ presentation.php >.Acceso: 31 jul. 2011.

BOYD, D. Digital audio and portable records. The basics. Oral History Association, [s.d.]. Disponible en: <http://www.oralhistory.org/technology/audio_basics >. Acceso: 31 jul. 2011. 
DARNTON, R. An early information society: new and media in the eighteenth-century Paris. The American Historical Review, v. 105, n. 1, 2000. Disponible en: <http://www. historycooperative.org/journals/ahr/105.1/ah000001.html>.Acceso: 31 jul. 2011.

LEE, J. K. Digital History in the History/Social Studies Classroom. The History Teacher, v. 35, n. 4, Aug. 2002. Disponible en: <http://www.historycooperative.org/journals/ht/35.4/ lee.html>. Acceso: 31 jul. 2011.

LINDSAY, E. Personal Blogs as Historical Documents. Genealogy Today, 21 Jan. 2009. Disponible en: $<$ http://www.genealogytoday.com/roots/xweb.mv?xc=RootsDocument\& $\mathrm{xo}=\mathrm{rescms} \& \mathrm{xr}=2718 \& \mathrm{xz}=\operatorname{artndx}>$. Acceso: 31 jul. 2011.

Resumen: A fines del siglo XX y principios del siglo XXI, los cambios tecnológicos que sientan las bases de la así denominada sociedad de la información ejercen una notable influencia en los estudios del pasado. La Historia Oral, en cuanto especialización disciplinaria, resulta afectada por estas innovaciones. Más específicamente, las clásicas rutinas del historiador oral se transforman gracias a dispositivos electrónicos que generan grabaciones digitales, programas informáticos que auxilian en el diseño, registro y procesamiento de entrevistas, y redes telemáticas que multiplican el acceso a fuentes históricas orales conservadas en repositorios virtuales. Por tal motivo, se requiere una evaluación global de las implicancias de estos cambios, a partir de la evidencia que aportan algunos ejemplos emblemáticos.

Palabras clave: Historia Oral, técnicas historiográficas, recursos digitales, heurística.

\title{
Oral History in digital contexts. Some recent transformations of a disciplinary subfield
}

\begin{abstract}
In the late twentieth and early twenty-first century, those technological changes that lay the groundwork for the so-called information society have a significant influence on the study of the past. Oral History, as a disciplinary subfield, it is affected by such innovations. More specifically, the oral historian's classic routines are modified by electronic devices that produce digital records, computational programs that assist in designing, recording and processing interviews, and computer networks that increases access to historical oral sources preserved in virtual repositories. For that reason, it is required an overall assessment of the implications of those changes, based on the evidence provided by some emblematic cases.
\end{abstract}

Keywords: Oral History, historiographical techniques, digital resourses, heuristics.

Recebido em $05 / 08 / 2011$

Aprovado em 12/10/2011 